\title{
Preliminary results of early clinical trials with the fully human anti-TNF $\alpha$ monoclonal antibody D2E7
}

\author{
Joachim Kempeni
}

Current pharmacological treatments for rheumatoid arthritis (RA), including non-steroidal anti-inflammatory drugs (NSAIDs), disease modifying anti-rheumatic drugs (DMARDs), and corticosteroids, have been moderately successful in alleviating the discomforts associated with swollen, painful joints. However, conventional medical approaches to treatment have had little or no impact on the disease course of RA. ${ }^{1}$ Innovative strategies, particularly those based on new concepts in the immunobiology of RA, are being developed to target cellular inflammatory mechanisms and potentially prevent disease progression. The more promising of these treatments seem to be those that block the effects of tumour necrosis factor (TNF) $\alpha$, because this proinflammatory cytokine seems to play a central part in the immunopathogenesis of RA. ${ }^{23}$

\section{Anti-TNF treatments}

Biological agents such as antibodies and soluble TNF receptors that bind TNF $\alpha$ with high specificity neutralise its activity and have been developed for use as therapeutic agents. Several are currently being evaluated in patients with RA (table 1). Infliximab, cA2, is a chimeric monoclonal antibody (mAb) that consists of the variable region of a murine anti$\mathrm{TNF} \mathrm{mAb}$ coupled to the constant region of human IgG1א. ${ }^{45}$ The resulting construct is approximately two thirds human. CDP571 is a humanised $\mathrm{mAb}$ consisting of the complementarity determining regions of a murine anti-TNF mAb grafted into a human immunoglobulin $(\operatorname{IgG} 4 \kappa){ }^{6}$ This $\mathrm{mAb}$ is approximately $95 \%$ human. Etanercept is a fusion protein consisting of two recombinant p75 TNF receptors attached to the $\mathrm{Fc}_{\mathrm{c}}$ portion of a human IgG1. ${ }^{7}$ Although this construct consists of two independent elements, which themselves contain 100\% human peptide sequences, they are arranged in an unnatural configuration.

The duration of the therapeutic efficacy of these TNF antagonists may be limited by an immune response to their non-human elements or artificially fused human sequences.

Correspondence to: Dr J Kempeni.

Table 1 Anti-TNF a treatments

\begin{tabular}{ll}
\hline Name & Description \\
\hline D2E7 & Fully human anti-TNF $\alpha$ mAb \\
CDP571 & Humanised anti-TNF $\alpha$ mAb (murine CDR) \\
Etanercept & $\begin{array}{l}\text { Fusion protein consisting of two recombinant p75 TNF } \alpha \\
\text { receptors and the Fc portion of human IgG1 } \\
\text { Chimeric murine/human anti-TNF- } \alpha \text { mAb }\end{array}$ \\
\hline
\end{tabular}

$\mathrm{TNF}=$ tumour necrosis factor; $\mathrm{mAb}=$ monoclonal antibody; $\mathrm{IgG}=$ immunoglobulin $\mathrm{G}$; $\mathrm{CDR}=$ complementarity determining region.
The development of antibodies to these biological agents could reduce their half life, thereby decreasing efficacy. ${ }^{8}$ In addition, an immune response could result in adverse events from the formation of immune complexes or the development of hypersensitivity. For these reasons, an antibody that is fully human may have greater therapeutic potential.

\section{Development of a fully human anti-TNF antibody}

BASF Pharma set out to develop a fully human, anti-TNF mAb structurally identical to naturally occurring human antibodies and therefore less likely to engender an immune response in the recipient. The generation of a fully human anti-TNF mAb required bioengineering techniques ${ }^{9}$ that mimic immune selection in humans and contrast with existing means of "humanising" murine monoclonal antibodies in that the antibodies derived are completely human. The result of this effort is D2E7, a new class of anti-TNF mAb, which may have advantages in minimising antigenicity in humans.

\section{Preclinical pharmacology}

The efficacy and safety of D2E7 were evaluated in a number of experimental systems. The ability of D2E7 to neutralise TNF bioactivity was demonstrated in three different in vitro cell systems. Additionally, the effectiveness of D2E7 in preventing polyarthritis was shown in a transgenic mouse model that mimics the clinical and histopathological progression of RA in humans. ${ }^{10}$ D2E7 treated mice showed no clinical signs of arthritis during the 11 week study period. Microscopical examinations of the ankle joints of the animals showed no histopathological changes. In contrast, control mice developed severe arthritis with cartilage destruction and bone erosion.

\section{Preliminary clinical data}

There are important similarities in the designs of the early clinical trials assessing D2E7 in RA. All studies enrolled patients with an established diagnosis of RA who also had active disease, as evidenced by having a combination of swollen and tender joints, increased concentrations of acute phase reactants, and prolonged early morning stiffness. In addition, all trials involved RA patients with long disease duration and a history of failure of several DMARDs. During the trials, patients were allowed to continue stable doses of NSAIDs and corticosteroids. Efficacy was assessed 
using composite criteria, such as the American College of Rheumatology improvement criteria (ACR 20) ${ }^{11}$ and the Disease Activity Score (DAS) ${ }^{12}$ Such criteria, which require improvement in multiple variables, are more stringent than is improvement in one or only selected clinical variables. For example, to be classified as a responder according to ACR 20 criteria, patients must demonstrate: (1) greater than or equal to $20 \%$ improvement in swollen joint count; (2) greater than or equal to $20 \%$ improvement in tender joint count and; (3) at least $20 \%$ improvement in three of five other measures (patient global assessment of disease activity, physician global assessment of disease activity, patient assessment of pain, an acute phase reactant (for example, erythrocyte sedimentation rate (ESR) or C reactive protein), and a measure of disability (for example, the Health Assessment Questionnaire)). The DAS is a composite score of tender joints, swollen joints, ESR and patient's assessment of disease activity as measured on a visual analogue scale.

Preliminary results of early trials of D2E7 are available (table 2). At the time of this review, some of the studies referenced have only been published in abstract form. In a randomised, double blind, placebo controlled phase I study, 120 patients were treated with single doses of D2E7 given in an ascending fashion ranging from 0.5 to $10 \mathrm{mg} / \mathrm{kg}$. ${ }^{13}$ Patients were treated in cohorts of 24 (18 patients were treated with D2E7 and six received placebo). After a wash out period of three weeks, the patients received a single dose of D2E7 or placebo by intravenous injection over 3-5 minutes. Patients were followed up for at least four weeks to determine the pharmacokinetics of D2E7, as well as to evaluate the safety and clinical efficacy of the compound in terms of onset, duration and magnitude of response. Positive response was defined as a decrease of at least 1.2 (compared with baseline) in the DAS. All parameters needed to calculate the response criteria as defined by the ACR 20 were also measured. Patients in whom the effect of D2E7 had declined below response status by week 4 entered an open label extension study. However, patients who maintained a response at week 4 were continued without retreatment until their response status was lost. Thereafter, these patients could also continue in the extension study.

The data from this first therapeutic trial in humans were very encouraging. In the three highest dose groups, $40-70 \%$ of patients achieved DAS and ACR 20 response status at 24 hours to 29 days of treatment. The therapeutic effects became evident within 24 hours to one week after D2E7 administration and reached the maximum effect after 1-2 weeks, with dose response reaching a plateau at $1 \mathrm{mg} / \mathrm{kg} \mathrm{D} 2 \mathrm{E} 7$. In contrast, only $19 \%$ of patients taking placebo achieved response status. Single doses of D2E7 were well tolerated and the dose increment scheme was followed as planned reaching the maximum dose of $10 \mathrm{mg} / \mathrm{kg}$ without any evidence of clinically relevant or dose related adverse effects. Pharmacokinetic parameters were calculated for a total of 89 patients from all dose groups. The systemic drug exposure (AUC) increased proportionally with increased dose. The mean total serum clearance was 0.180 to $0.271 \mathrm{ml} / \mathrm{min}$, and the steady state volume of distribution ranged from 0.063 to $0.0761 / \mathrm{kg}$ indicating that distribution of D2E7 was mostly in the intravascular space. The estimated mean terminal half life was 11.6 to 13.7 days.

Patients who participated in the open label extension study received a second blinded dose identical to their first dose (medication was given after a minimum period of four weeks and only after loss of their initial response status)..$^{14}$ From the third dose onwards, all patients were given active drug (that is, the placebo patients received D2E7 doses according to their dose group). D2E7 was administered every two weeks until responses could be rated as "good", defined as an absolute DAS of < 2.4. To measure the duration of the good response, these patients were retreated only upon disease flare up. To keep as many patients as possible in the study for the long term evaluation of safety, patients who did not respond well after 0.5 or $1 \mathrm{mg} / \mathrm{kg}$ received higher doses of up to a maximum of $3 \mathrm{mg} / \mathrm{kg}$. Doses were kept constant in the patients on 3,5 or 10 $\mathrm{mg} / \mathrm{kg}$. Treatment lasting several years is intended. The response in the DAS over time demonstrated sustained therapeutic effects and some continuing improvement after multiple infusions of D2E7. Response rates of more than $80 \%$ have been achieved with a mean dosing interval of 2.5 weeks. After six months, $86 \%$ of patients continued to receive treatment with D2E7 indicating that long term intravenous treatment with D2E7 in the dose range from 0.5 to $10 \mathrm{mg} / \mathrm{kg}$ was well tolerated.

The safety and efficacy of weekly subcutaneous administration of $0.5 \mathrm{mg} / \mathrm{kg} \mathrm{D} 2 \mathrm{E} 7$ was evaluated in 24 patients with active RA in a phase I placebo controlled trial. ${ }^{15}$ After a wash

Table 2 Early trials of D2E7 in rheumatoid arthritis

\begin{tabular}{|c|c|c|c|c|}
\hline Trial design & Patients & D2E7 dosing schedule & $\begin{array}{l}\text { Concurrent } \\
D M A R D\end{array}$ & $\begin{array}{l}\text { Maximum } \\
\text { ACR } 20 \\
\text { response }\end{array}$ \\
\hline Randomised, double blind, placebo controlled & $\begin{array}{l}\mathrm{n}=120 ; 83 \% \mathrm{RF}^{+} ; \text {mean disease } \\
\text { duration }=12 \text { years; mean DMARDs } \\
\text { failed }=3.6\end{array}$ & $\begin{array}{l}\text { Single and multiple iv injections, } \\
\text { ascending doses ranging from } \\
0.5 \text { to } 10 \mathrm{mg} / \mathrm{kg}\end{array}$ & No & $78 \%$ \\
\hline Randomised, double blind, placebo controlled & $\begin{array}{l}\mathrm{n}=24 ; \text { mean disease duration }=10 \text { years; } \\
\text { mean DMARDs failed }=3.4\end{array}$ & Weekly $0.5 \mathrm{mg} / \mathrm{kg}$ sc injections & No & $70 \%$ \\
\hline Randomised, double blind, placebo controlled & $\begin{array}{l}\mathrm{n}=54 ; 87 \% \mathrm{RF}^{+} ; \text {mean disease } \\
\quad \text { duration }=11 \text { years; mean DMARDs } \\
\text { failed (including MTX) }=3.6\end{array}$ & Single iv or sc injection of $1 \mathrm{mg} / \mathrm{kg}$ & MTX & $67 \%$ * \\
\hline
\end{tabular}

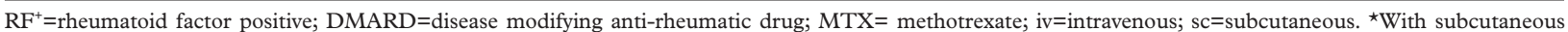
administration. 
out period of three weeks, patients were treated with subcutaneous D2E7 or placebo for three months. The dose of D2E7 was increased to 1 $\mathrm{mg} / \mathrm{kg}$ subcutaneously weekly for nonresponders or those losing their responder status. Blood samples were collected to determine D2E7 plasma concentrations. All responding patients continued in an open label extension of this study. Based on preliminary data, plasma concentrations of D2E7 after multiple subcutaneous doses were comparable to those achieved with intravenous administration. Up to $78 \%$ of patients achieved a DAS/ACR 20 response after three months of treatment with subcutaneous D2E7. With the exception of mild and transient injection site reactions, adverse events occurred with the same frequency and distribution in the D2E7 and placebo groups. The investigators concluded that D2E7 given subcutaneously was safe and as effective as when administered intravenously demonstrating that subcutaneous self administration is a promising approach for D2E7 delivery.

Monotherapy is often inadequate to control arthritic symptoms and rapid progression of RA. D2E7 (1 mg/kg as a single subcutaneous or intravenous injection) was evaluated in a randomised, double blind, placebo controlled trial in patients whose stable dose of methotrexate was insufficient to control symptoms. An ACR 20 response was seen in $67 \%$ and $72 \%$ of patients receiving D2E7 by subcutaneous and intravenous injection, respectively. The safety profile of single dose D2E7 administration was comparable to that of placebo.

Collectively, these early data suggest that the fully human anti-TNF $\alpha \mathrm{mAb}$ D2E7 is safe and effective as monotherapy or in combination with methotrexate when administered by single and multiple intravenous and subcutaneous injections. Additional studies are underway to further define optimal use of this novel treatment.

1 Brooks P. Clinical management of rheumatoid arthritis. Lancet 1993;341:286-90.

2 Maini R, Brennan F, Williams R, et al. TNF-a in rheumatoid arthritis and prospects of anti-TNF therapy. Clin Exp Rheumatol 1993;11 (suppl 8):S173-5.

3 Feldmann M, Brennan F, Chu C, et al. Does TNF-a have a pivotal role in the cytokine network in rheumatoid arthritis? In: Friers W, ed. Tumor necrosis factor: molecular and cellular biology and clinical relevance. Basel: Karger, 1993:144-52.

biology and clinical relevance. Basel: Karger, 1993:144-52.
4 Elliott MJ, Maini RN, Feldmann M, et al. Treatment of rllott $\mathrm{MJ}$, Maini $\mathrm{RN}$, Feldmann $\mathrm{M}$, et al. Treatment of
rheumatoid arthritis with chimeric monoclonal antibodies to tumor necrosis factor alpha. Arthritis Rheum 1993;36: to tumor 90 .

5 Scallon B, Moore M, Trinh H, Knight D, Ghrayeb J. Chimeric anti-TNF-alpha monoclonal antibody cA2 binds recombinant transmembrane TNF-alpha and activates immune effector functions. Cytokine 1995; 7:251-9.

6 Rankin EC, Choy EH, Kassimos D, et al. The therapeutic effects of an engineered human anti-tumour necrosis factor alpha antibody (CDP571) in rheumatoid arthritis. Br J Rheumatol 1995; 34:334-42.

7 Moreland LW, Baumgartner SW, Schiff MH, et al. Treatment of rheumatoid arthritis with a recombinant human tumor necrosis factor receptor (p75)-Fc fusion protein. N Engl J Med 1997;337:141-7.

8 Kavanaugh AF. Anti-tumor necrosis factor-alpha monoclonal antibody therapy for rheumatoid arthritis. Rheum Dis Clin North Am 1998;24:593-614.

9 Jespers L, Roberts A, Mahler S, Winter G, Hoogenboom H. Guiding the selection of human antibodies from phage display repertoires to a single epitope of an antigen. Biotechnology (NY) 1994;12:899-903.

10 Keffer J, Probert L, Cazlaris $\mathrm{H}$, et al. Transgenic mice expressing human tumour necrosis factor: a predictive genetic model of arthritis. EMBO J 1991;10:4025-31.

11 Felson D, Anderson J, Boers M, et al. The American College of Rheumatology preliminary core set of disease activity measures for rheumatoid arthritis clinical studies. Arthritis Rheum 1995;38:727-35

12 van Gestel A, Prevoo M, Van'T Hoff M, et al. Development and validation of the European League Against Rheumaism response criteria for rheumatoid arthritis. Arthritis Rheum 1996;39:34-40.

13 van de Putte L, van Riel P, den Broeder A, et al. A single dose placebo-controlled phase I study of the fully human anti-TNF antibody D2E7 in patients with rheumatoid arthritis. Arthritis Rheum 1998;41 (suppl):57.

14 Rau R, Sander O, den Broeder A, et al. Long term efficacy and tolerability of multiple iv doses of the fully human antiTNF antibody D2E7 in patients with rheumatoid arthritis. Arthritis Rheum 1998;41 (suppl):55.

15 Schattenkirchner M, Krüger K, Sander O, et al. Efficacy and tolerability of weekly subcutaneous injections of the fully human anti-TNF antibody D2E7 in patients with rheumatoid arthritis. Arthritis Rheum 1998;41 (suppl):57. 